\title{
COMPARATIVE ANALYSIS OF PHYSICAL CONDITIONS IN THE SOLAR AND PROCYON ATMOSPHERES
}

\author{
I.N. ATROSHCHENKO, A.S. GADUN, R.I. KOSTIK, K.N. PIKALOV \\ Main Astronomical Observatory \\ Academy of Sciences of the Ukrainian SSR \\ 252127, Kiev, USSR
}

\begin{abstract}
An analysis is made of the velocity field determined from spectral absorption line profiles as well as inhomogeneous element sizes obtained from stochastic theory. The results of three-dimensional simulations of convective motions in the photospheres of the Sun and Procyon are presented. It is concluded that penetrative convection covers a considerable fraction of the Procyon photosphere.
\end{abstract}

Velocity fields in the solar and Procyon photospheres. Inhomogeneous element sizes obtained from spectral line profiles

Equivalent widths and central depths of selected lines of neutral iron have been determined from an atlas of the Sun as a star (Kurucz et al., 1984) and the Procyon atlas (Griffin and Griffin, 1979). Spectral line profiles from the Procyon atlas were corrected for instrumental distortion in accordance with data given by Mackle et al. (1975).

The turbulence parameters were determined together with the iron abundance by a method of Gurtovenko and Kostik (1989). The calculations were performed with the SPANSAT program (Gadun and Sheminova, 1988). The Holweger-Müller model (1974) was used for the Sun, the Steffen (1985) model for Procyon with the parameters $T_{\text {eff }}=6500$ and $\log g=4.04$, and with solar abundances. Rotation was taken into account through direct averaging of the intensity profiles over the disk. Effective line formation depths were calculated using the depression contribution functions.

The sizes of the photospheric inhomogeneities were estimated using stochastic line formation theory (the Uhlenbeck-Ornstein process), which contains two free parameters: $L$, the element size, and $\xi$, the RMS velocity, where $\xi^{2}=V_{m i}^{2}+V_{m a}^{2}$. Using known chemical abundances, $L$ can then be determined by fitting theoretical equivalent widths and central depths with corresponding observed values.

Fourty-five neutral iron lines with empirical oscillator strengths and excitation potentials of the lower level from 0.05 to $2.61 \mathrm{eV}$ were used. The iron abundance was found to be $7.64 \pm 0.3$ in the solar and 7.53 \pm 1.0 in the Procyon photosphere. The dependence of $V_{m i}$, $V_{m a}$, and $L$ on geometrical height are given in Figures 1 and 2. It is seen from these figures that

421

J. O. Stenflo (ed.), Solar Photosphere: Structure, Convection, and Magnetic Fields, 421-425.

(c) 1990 by the IAU. 


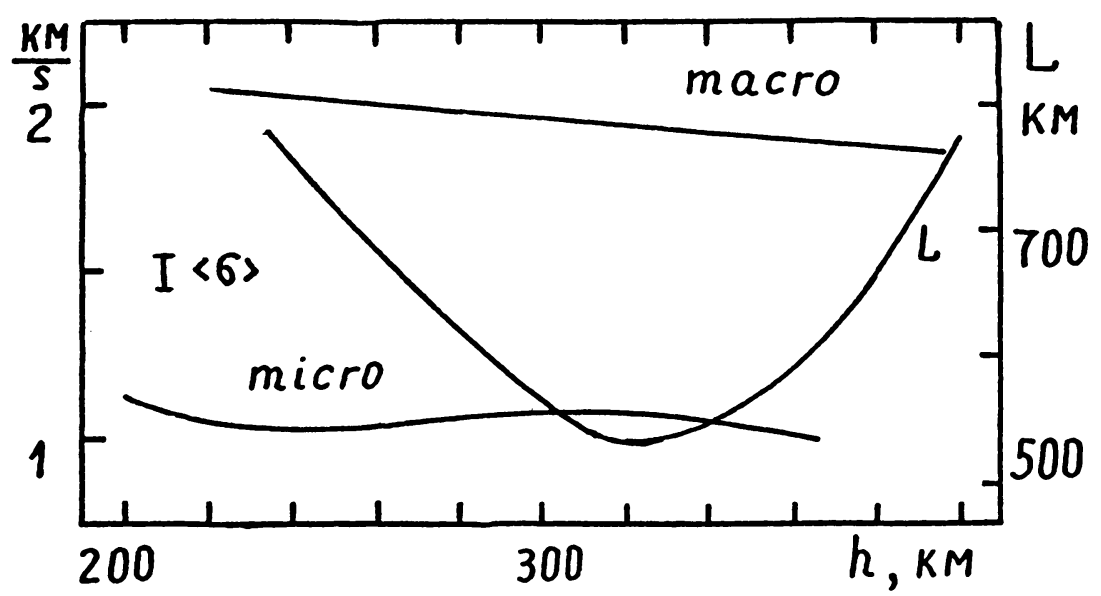

Figure 1. Dependence of $V_{m i}, V_{m a}$, and $L$ on geometrical height $h$ in the solar photosphere.

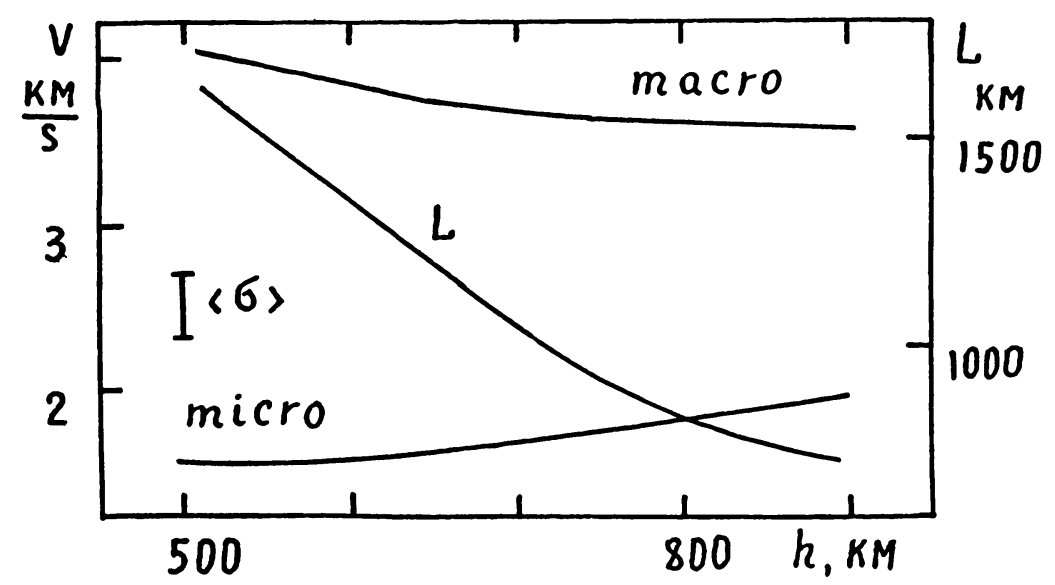

Figure 2. Dependence of $V_{m i}, V_{m a}$, and $L$ on geometrical height $h$ in the Procyon photosphere.

- the amplitude of the general velocity field in the Procyon photosphere is twice as large as in the solar photosphere;

- the microturbulent velocity in the Procyon photosphere increases with height but with a local decrease at a height of about $300 \mathrm{~km}$. The solar microturbulent velocity decreases with height;

- the behaviour of $L$ in the solar photosphere can be interpreted as follows. Three regions can be identified in the solar photosphere: the penetrative convection region $(h<310$ $\mathrm{km})$, the intermediate region $(h=310-370 \mathrm{~km})$, and the oscillation region $(h>370$ $\mathrm{km})$. Their heights are determined from the observed spectrum of the Sun as a star, since they differ from those for the disk centre (Atroshchenko et al., 1989a). In the case of Procyon penetrative convection is the dominating feature (Figure 2); 
- the region where $V_{m i}$ increases coincides with that where the element sizes decrease. Thus an increase of $V_{m i}$ with height may indicate the existence of fragmentation processes of vortices in the Procyon photosphere.

General results of direct numerical simulations of convective motions in the solar and Procyon photospheres

The ideas and computational schemes have been described by Gadun (1986) and Atroshchenko et al. (1989b) in detail. The main results are shown in Figures $3-6$. The following conclusions may be made:

1. Convective motions penetrate into the stable solar photospheric layers, to heights of $200-300 \mathrm{~km}$. In the Procyon case penetrative convection covers virtually the entire computational region (up to $1000 \mathrm{~km}$, from $\tau_{5}=1$ ).

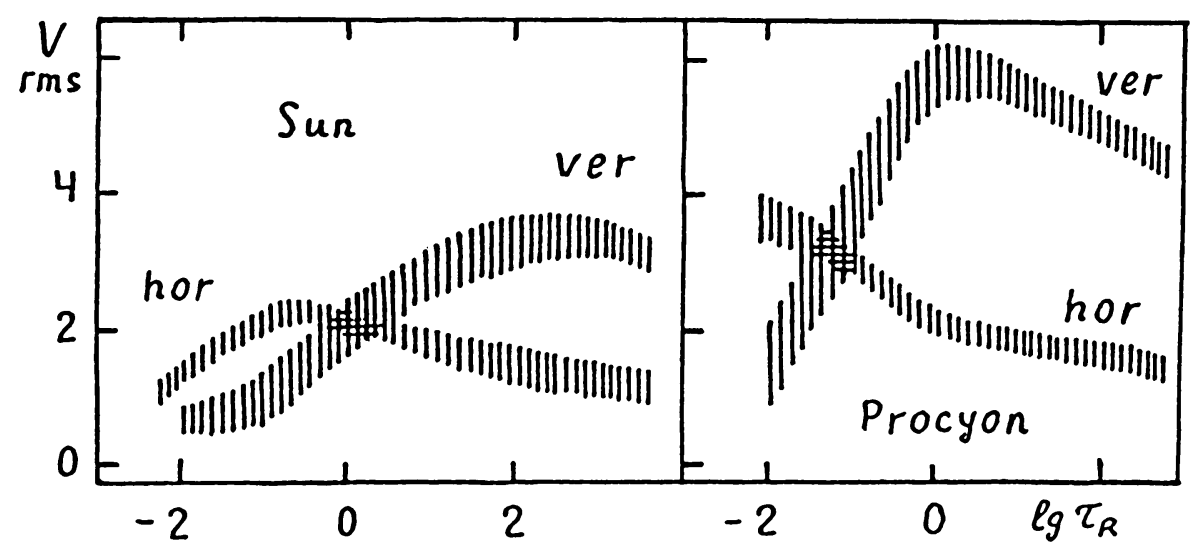

Figure 3. Calculated velocities in the solar and Procyon envelopes.

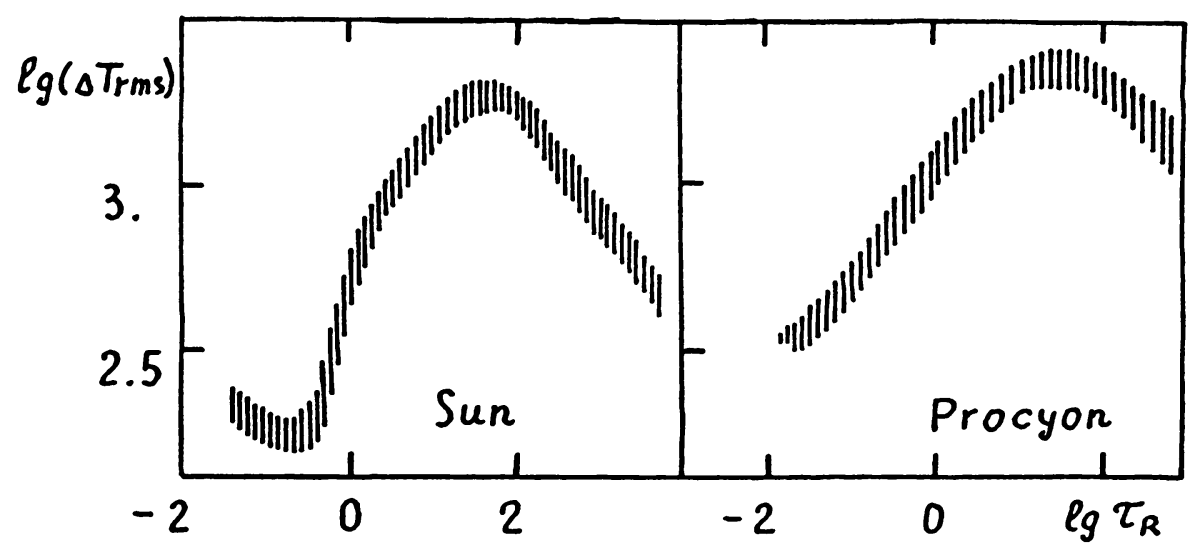

Figure 4. RMS temperature fluctuations from the three-dimensional inhomogeneous solar and Procyon models. 
2. Calculated convective velocities in the Procyon photosphere are found to be twice as large as in the Sun.

3. The temperature fluctuations for the Sun and Procyon are similar, but they decrease slower with height in the Procyon photosphere.

4. As a consequence, the intensity fluctuations in the Procyon photosphere are higher than for the Sun.

5. The greater penetration of hot elements into the stable Procyon layers is the cause of the inverse limb-effect behaviour as compared with the solar case.

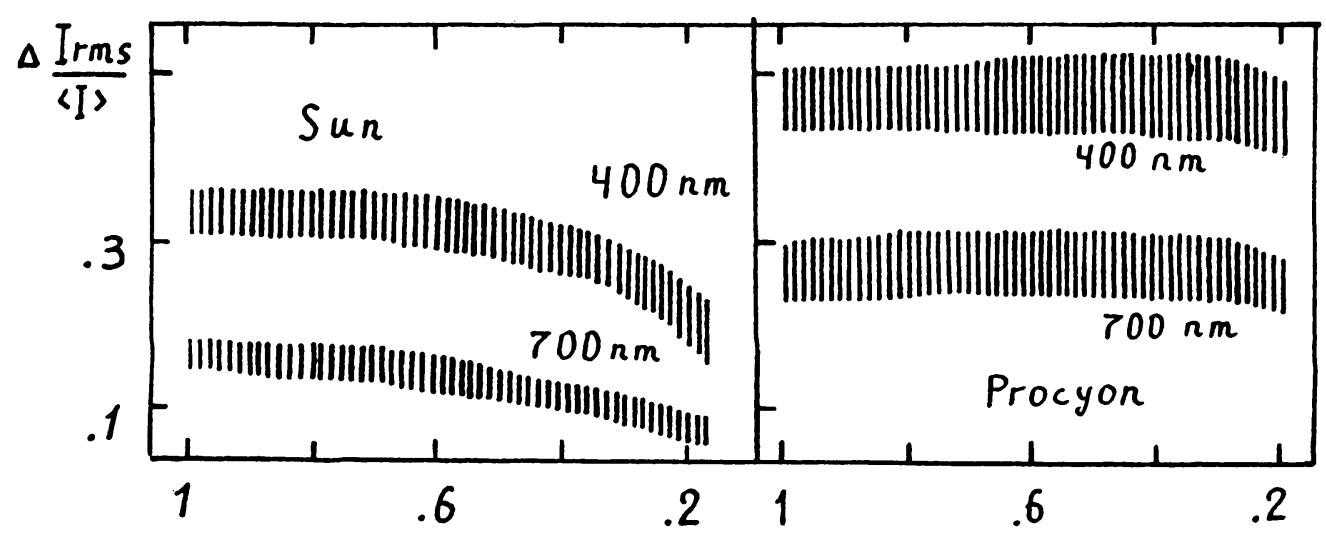

Figure 5. Relative RMS intensity fluctuations from the three-dimensional inhomogeneous models.

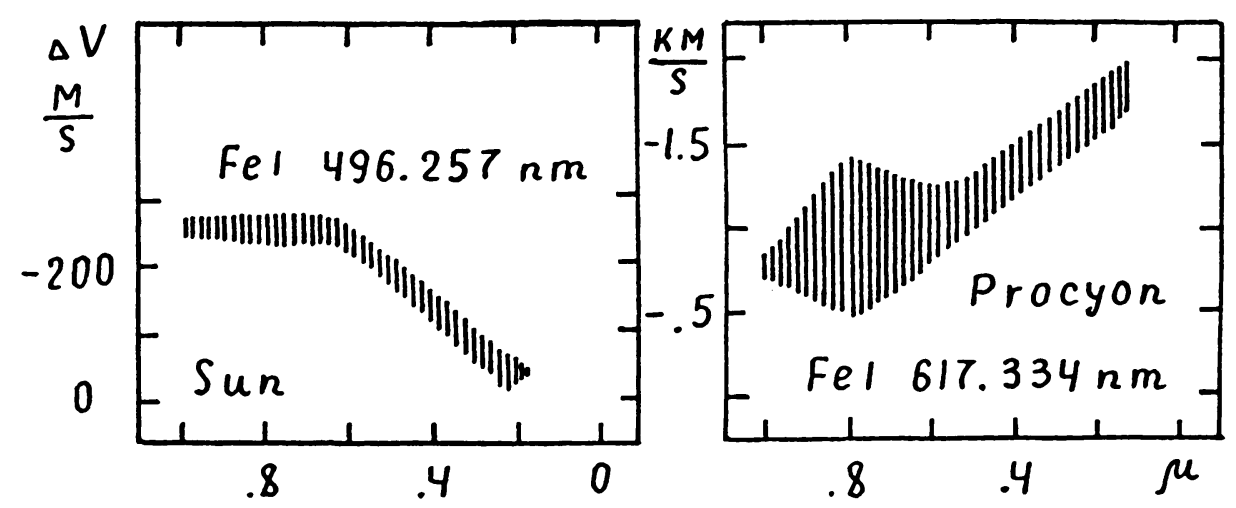

Figure 6. The limb-effect calculated from three-dimensional models. 
Accordingly penetrative convection plays a considerably larger role in the photosphere of Procyon than in the Sun.

\section{References}

Atroshchenko, I.N., Gadun, A.S., Kostik, R.I. (1989a) Solar and Stellar Granulation, NATO ASI Series C 263, 135.

Atroshchenko, I.N., Gadun, A.S., Kostik, R.I. (1989b) Solar and Stellar Granulation, NATO ASI Series C 263, 521.

Gadun, A.S. (1986) Preprint ITP of Ac. Sc. Ukr. SSR, ITP-86-106R, Kiev.

Gadun, A.S., Sheminova, V.A. (1988) 'SPANSAT: Program for LTE Calculations of Absorbtion Line Profiles in Stellar Atmospheres', Preprint ITP-88-87R, Kiev.

Griffin, R., Griffin, R. (1979) A Photometric Atlas of the Spectrum of Procyon 3140 - 7470 $\AA$, Cambridge, UK.

Gurtovenko, E.A., Kostik, R.I. (1989) Fraunhoferov Spektr i Sistema Sil Oscillatorov 44, Kiev.

Holweger, H., Müller, E.A. (1974) Solar Phys. 39, 19.

Kurucz, R.L., Furenlid, I., Brault, J., Testerman, L. (1984), Solar Flux Atlas from 290 to $1300 \mathrm{~nm}$, Harvard University.

Mackle, R., Griffin, R., Griffin, R., Holweger, H. (1975) Astron. Astrophys. Suppl. Ser. 19, 303.

Steffen, M. (1985) Astron. Astrophys. Suppl. Ser. 59, 403. 